\title{
JOHN STUART MILL Y EL PELIGRO DEL ESTANCAMIENTO EN LAS SOCIEDADES MODERNAS
}

\author{
María Pollitzer \\ Universidad Católica Argentina
}

\begin{abstract}
Resumen: A lo largo de su vida John Stuart Mill no dejó de alertar a sus contemporáneos sobre un fenómeno que consideraba verdaderamente peligroso e inclusive como el mal principal que comenzaba a aquejar a las sociedades modernas: el estancamiento o la inmovilidad. Estrechamente vinculado con muchas de las ideas-eje de la reflexión milliana, no obstante el peligro del estancamiento no ha sido analizado en detalle. El siguiente artículo tiene por objeto, por consiguiente, detener la mirada sobre este fenómeno puntual, y analizar tanto su naturaleza como los factores que contribuyen a su aparición y sus posibles efectos.
\end{abstract}

Palabras clave: estancamiento, John Stuart Mill, civilización, antagonismo, uniformidad.

Recibido: agosto 2012; aceptado: octubre 2012;

María Pollitzer. Doctora en Ciencias Políticas, Universidad Católica Argentina (UCA). Profesora adjunta en las cátedras de historia de las ideas políticas, teoría política y metodología de la investigación histórica, UCA. Dirección electrónica: maria_pollitzer@hotmail.com. 


\section{JOHN STUART MILL AND HIS WARNING ABOUT STAGNATION IN MODERN SOCIETIES}

Abstract: Throughout his life, John Stuart Mill warned contemporaries about stagnation or immobility, a most dangerous phenomenon, perhaps the chief evil then begining to afflict modern societies. Despite its close links with many other key ideas in Mill, the perils of stagnation have not yet been subject to detailed scrutiny. This paper offers an analysis of the nature and sources of stagnation as well as its likely effects.

Key words: stagnation, John Stuart Mill, civilization, antagonism, uniformity.

Received: August 2012; accepted: October 2012.

\section{Introducción}

Com omo tantos otros pensadores del siglo XIX, John Stuart Mill hizo suyo el desafío de sondear la naturaleza de la sociedad moderna. Convencido de que no era posible "hacer que el río [volviera] sobre su curso", de que el avance de la democracia era un hecho incontestable y que el triunfo de las clases medias parecía irreversible, sus esfuerzos se concentraron en descifrar esta "nueva" sociedad para descubrir en ella sus potencialidades $\mathrm{y}$, al mismo tiempo, advertir a sus connacionales acerca de los "riesgos y precipicios" que los amenazaban".

El objetivo de este trabajo es analizar, precisamente, uno de los "riesgos" que llamó la atención de Mill y al que él mismo identificó en varias oportunidades como "el más grande", "el verdadero y principal peligro para la democracia", e incluso como "la fuente primera de casi todos los males sociales" 3 . Me refiero puntualmente al estancamiento. En efecto, las denuncias sobre este mal aparecen tempranamente en los escritos del inglés, tanto en sus discursos juveniles como en varios de

1 John Stuart Mill, Considerations on Representative Government, en The Collected Works of John Stuart Mill, J. M. Robson (ed.) (1963-1991), Vol. XIX, p. 380, en adelante $C W$. p. 231).

${ }^{2}$ Cfr. "The Spirit of the Age. I", Examiner (9-1-1831) (CW Vol. XXII,

3 Estas expresiones aparecen en las cartas enviadas a Ralph Waldo Emerson el 12-8-1867 ( $C W$, Vol. XVI, p. 1306) y a John Appleton el 24-9-1863 ( $C W$, Vol. XV, pp. 647-648). 
sus artículos periodísticos. También se asoman repetidas veces en sus grandes obras, publicadas en su etapa de madurez, y se reiteran en su correspondencia privada ${ }^{4}$. Tan sólo a título ilustrativo, basta con recordar aquella carta dirigida a su amigo Alexis de Tocqueville en mayo de 1840 en la que, una vez concluida la lectura del segundo volumen de Democracia en América, Mill confiesa que considera "un cumplido a la justeza de sus propias opiniones", el hecho de haber comprobado que una de las conclusiones a las que había arribado el viajero francés coincidía "exactamente" con lo que él mismo venía pensando desde hacía varios años de manera solitaria en las tierras británicas. Ello es,

que el verdadero peligro para la democracia, el mal verdadero contra el que hay que luchar, empleando todos los recursos humanos, mientras no sea demasiado tarde para que no sean sino suficientes para contenerlo, no es la anarquía ni el amor al cambio, sino el estancamiento y la inmovilidad a la china ${ }^{5}$.

${ }^{4}$ Cfr. a modo de ejemplo: "The Universities" ( $C W$, Vol. XXVI, p. 349); "French News", Examiner (13-2-1831), (CW, Vol. XXII, p. 263); "State of Parties in France", Examiner (28-8-1831), (CW, Vol. XXIII, pp. 336-341); "French and English Journals", Examiner (2-12-1832), (CW, Vol. XXIII, p. 530); "French News", Examiner (26-1-1834), (CW, Vol. XXIII, p. 671); "Bentham", London \&Westminster Review (agosto 1838), (CW, Vol. X, p. 108); "De Tocqueville on Democracy in America. II", Edinburgh Review (octubre 1840), (CW, Vol. XVIII, pp. 188, 196) y "Guizot's Essays and Lectures on History", Edinburgh Review (octubre 1845), (CW, Vol. XX, p. 270). En el capítulo V del libro V de Principles of Political Economy Mill vincula la falta de discusión con el estancamiento intelectual; en el capítulo III de On Liberty, examina la relación entre el estancamiento y el espíritu de individualidad; y en el capítulo VII de Considerations on Representative Government argumenta que éste aparece cuando cesa el conflicto y cuando la sociedad carece de un punto de apoyo para la resistencia individual frente al poder gobernante.

${ }^{5}$ Carta enviada a Alexis de Tocqueville el 11-5-1840 ( $\mathrm{CW}$, Vol. XIII, pp. 433-435). [El resaltado es mío] A renglón seguido, Mill agrega: “Al encontrar que esta opinión sobre el asunto se ha presentado con la misma fuerza de evidencia a vos, que sois la más alta autoridad viva (...) en el asunto, en adelante la consideraré como una verdad científicamente establecida y la defenderé contra viento y marea con decuplicada pertinacia”. Este juicio que Mill realiza en un ámbito privado lo reitera a los pocos meses con ocasión de la reseña que publica sobre el segundo volumen de la Democracia en América. Lo que a ambos preocupa "tanto en el gobierno como en el intelecto y la moral, no es demasiada libertad sino la muy rápida sumisión; no la anarquía sino la servidumbre; no el cambio demasiado rápido sino el estancamiento chino". ("De Tocqueville on Democray in America. II", CW, Vol. XVIII, p. 188). 
Como puede observarse, lo que parece preocupar a Mill no es el movimiento y la agitación constante con la que en principio se identificaba a la sociedad civilizada (o democrática, en términos tocquevillianos). No es tampoco la perspectiva de una suerte de anarquía intelectual, producto de la diversidad de ideas y sentimientos que los hombres modernos profesan, guiados por un deseo inmoderado de autonomía. $\mathrm{Ni}$ acaso, la posibilidad de que la sociedad se vea convulsionada y amenazada por la irrupción de doctrinas "peligrosas" (léase, socialistas ${ }^{6}$ ). Por el contrario, su gran temor resulta de aquello que observa a su alrededor: una sociedad que se encamina lentamente hacia un escenario de inmovilidad y estancamiento en el que el progreso y la libertad se ven seriamente amenazados.

El tenor de este tipo de afirmaciones abre una serie de interrogantes que parecen estar ausentes en la vasta literatura que rodea la obra de este autor ${ }^{7}$. Cabría preguntarse, por ejemplo, ¿cuál es, para Mill, la naturaleza de este peligro particular?, ¿cuáles son las raíces con las que lo identifica?, ¿cuáles, sus implicancias?, o bien, ¿sobre qué escenarios se proyecta? Este tipo de precisiones son necesarias para comprender la importancia y centralidad que él asigna a este problema en el conjunto de su obra. Sobre ellas se estructura el análisis que sigue a continuación.

\section{El estancamiento, un mal que se conjuga en plural}

Comencemos por el principio. ¿A qué se refiere Mill cuando habla de estancamiento o inmovilidad "a la china”? ¿Qué tipo de peligro es el que visualiza? Arribar a una definición exacta sobre la naturaleza de este peligro no resulta una tarea sencilla. Entre otras razones, porque Mill no dejó ningún libro ni artículo en el que examinara esta cuestión de manera sistemática. Por consiguiente, resulta inevitable atender a las reflexiones que el autor brinda no sólo en sus obras canónicas, sino también en sus artículos periodísticos, reseñas bibliográficas, inter-

${ }^{6}$ Cfr. "French News", Examiner (26-1-1834), (CW, Vol. XXIII, p. 671).

${ }^{7} \mathrm{Si}$ bien es cierto que muchos de los tópicos vinculados al estancamiento han recibido gran atención de parte de los estudiosos de Mill, hasta donde sé la problemática puntual del estancamiento no ha sido tematizada directamente en cuanto tal. 
venciones parlamentarias y en su abundante correspondencia. Por otra parte, si bien las referencias al estancamiento son recurrentes en el conjunto de su obra, ellas suelen aparecer de forma aislada y escueta. En varias ocasiones, incluso, este "cruzado solitario" 8 se vale de metáforas o comparaciones para graficar la "enfermedad" que denuncia. En virtud de ello, quizás lo más adecuado sea describirlo con ayuda de las notas distintivas con las que él mismo lo asocia.

Lo primero que se desprende de su diagnóstico es la idea de que éste es un mal que puede anidar tanto en el mundo político como en la esfera moral e intelectual. Una sociedad cuya vida política está estancada es una sociedad en la que la política ha caído en un inquietante reposo, una sociedad que se halla desprovista de dinamismo interno, que ha perdido vitalidad y carece de movimiento. Se trata de una sociedad que prioriza el orden, la conservación de lo adquirido y la aparente estabilidad por sobre el cambio y el progreso; que teme las innovaciones y recela de las tensiones. Una sociedad, en fin, que avala el curso adoptado por aquellos pocos que detentan el poder y que han cerrado filas en torno a él. En ella imperan, por consiguiente, la rutina y el mantenimiento del statu quo ${ }^{9}$. Este tipo de inmovilidad, que semeja un estado de ensueño o semi-vigilia, convierte a la sociedad en un terreno propicio para el advenimiento del despotismo (ya sea en su versión cesarista como bajo su rostro moderno, el despotismo de tipo administrativo que ejerce un poder centralizado y tentacular ${ }^{10}$ ). No se confunde con él, pero ciertamente lo favorece: un pueblo adormecido se deja arrebatar fácilmente la libertad, en tanto que carece de las disposiciones necesarias para retenerla. Dicho en otros términos, el estancamiento político es la antesala de nuevas formas de opresión política.

8 El calificativo es de Stefan Collini (Public Moralists: Political Thought and Intellectual Life in Great Britain 1850-1930, 1991, p. 129), quien sostiene que Mill se encontraba a gusto presentándose como un "cruzado solitario", carente de un ejército en el cual apoyarse y movido tan sólo por aquel incentivo que proviene de la rectitud de la causa a la que se sirve.

${ }^{9}$ Cfr. "French News", Examiner (13-2-1831), (CW, Vol. XXII, p. 263) y "State of Parties in France", Examiner (28-8-1831), (CW, Vol. XXIII, pp. 336$341)$.

${ }^{10}$ Cfr., especialmente, “Centralisation”, Edinburgh Review (abril 1862), ( $C W$, Vol. XIX) y Considerations on Representative Government ( $C W$, Vol. XIX, p. 400). 
Por su parte, el estancamiento moral e intelectual es caracterizado por Mill como un estado de "pacificación intelectual"11 que se encuentra signado por la ausencia de grandes controversias, la escasez de convicciones fuertes y pasiones elevadas; un estado en el que el espíritu humano se rebaja, se embota, se atrofia y en el que prima una creciente uniformidad en materia de ideas, sentimientos, gustos y hábitos. Es famoso aquel pasaje de On Liberty en el que constata que todos a su alrededor "leen los mismos libros, escuchan las mismas cosas, ven las mismas cosas, van a los mismos lugares, tienen sus deseos y sus miedos dirigidos a los mismos objetos, tienen los mismos derechos y libertades y las mismas maneras de hacerlos valer"12. Este tipo de estancamiento también supone un estado de mediocridad y dogmatismo en el que tanto el desarrollo de la inteligencia como el cultivo de la individualidad implican una verdadera hazaña ${ }^{13}$. Sobre semejante escenario, además, se erige lentamente un nuevo actor social capaz de oprimir y cercenar las libertades individuales: la opinión pública. Su imperio da lugar a lo que él denomina "la tiranía de la opinión", aquella que no mata ni ataca directamente el cuerpo de los hombres sino que va derecho al alma y "la esclaviza". Despotismo y estancamiento forman parte de un círculo vicioso que se retroalimenta y que se traduce, en definitiva, en el "sacrificio del coraje moral del espíritu humano"14.

Identificados los tipos de estancamiento que Mill percibe, resulta conveniente avanzar un poco más en el análisis y examinar cuáles son los escenarios sobre los que éste se despliega.

\section{Estancamiento y movimiento en los períodos inorgánicos o de transición}

Una lectura transversal de su obra indica que el estancamiento es percibido como un peligro actual y no sólo potencial, que afecta tanto

11 On Liberty (CW, Vol. XVIII, p. 242).

12 On Liberty ( $\mathrm{CW}$, Vol. XVIII, p. 274).

${ }^{13}$ Mill comparte la desolación que le provoca estar frente a un mundo que se encamina hacia la nivelación de espíritus con su amigo, el historiador T. Carlyle: "A veces pienso que en lugar de montañas y valles, el dominio del intelecto está por convertirse en una llanura muerta, nada muy por encima del nivel general, nada muy por debajo suyo" (carta fechada el 17-7-1832, CW, Vol. XII, p. 112). En "Notes on the Newspapers" advierte que en su época, "tener carácter es ya una considerable distinción, y tener el coraje para actuar a su altura, algo extraordinario" ( $C W$, Vol. VI, p. 173).

14 Las expresiones están tomadas de On Liberty ( $C W$, Vol. XVIII, pp. 323 у 242). 
a Inglaterra como a Francia. Ahora bien, ¿cómo se conjuga esta afirmación con la idea, también presente al menos en los primeros escritos de Mill, de que la sociedad que lo rodea se encuentra atravesando un período de transición en el que la nota saliente pareciera ser, paradójicamente, el cambio o la mudanza que se observan por doquier? ${ }^{15}$

Como es bien sabido, a los pocos años de su famosa crisis intelectual Mill comenzó a interesarse por las ideas profesadas por los saintsimonianos. Su principal interlocutor fue Gustave d'Eichthal, quien a fines de la década del veinte se trasladó a Londres junto con Charles Duveyrier. También conoció a Enfantin y llegó a afirmar que leía "todo" lo que escribían estos pensadores ${ }^{16}$. Se sentía atraído particularmente por el lugar que aquellos otorgaban a los sectores ilustrados dentro de la sociedad; su preocupación por la pobreza y su llamado a revisar el concepto de propiedad; su apoyo a la emancipación femenina y, finalmente, por la manera en la que esta escuela leía la historia, convencida de que toda filosofía política suponía una determinada teoría sobre el progreso humano. Tal como recuerda en su Autobiography, este último fue uno de los puntos que llamó particularmente su atención. Los saint-simonianos enseñaban que la historia se encontraba signada por períodos orgánicos o naturales y períodos inorgánicos o de transición. En los primeros, el poder temporal y la influencia moral son ejercidos sin discusión por las personas más capacitadas que la sociedad encuentra y el pueblo, por lo general, respeta las leyes y las instituciones que rigen su vida sin cuestionarlas. Por contrapartida, en los períodos de transición, la unión entre el poder temporal y quienes ejercen la influencia moral en la sociedad se rompe. La autoridad que fija las opiniones y los sentimientos de quienes aún no están suficientemente acostumbrados a pensar por sí mismos no reside en las mentes más cultivadas y, al no existir doctrinas establecidas, el mundo de las opiniones es un "mero caos".

Imbuido en este marco conceptual, Mill publicó (a comienzos de 1831) una serie de seis artículos en el periódico semanal de tendencia radical, Examiner, bajo el título de "The Spirit of the Age". En ellos aventuró un primer diagnóstico sobre la realidad de su época a la que, aplicando las categorías recién mencionadas, no dudó en calificar de

${ }^{15}$ Cfr. "The Spirit of the Age. I" ( $C W$, Vol. XX, p. 228 y "Civilization", London and Westminster Review (abril 1836), (CW, Vol. XVIII, p. 126).

${ }^{16}$ Autobiography (CW, Vol. I, p. 173). 
transitoria ${ }^{17}$. Allí constata que sus contemporáneos consideran inadecuadas y perimidas las instituciones y las doctrinas que hasta entonces los habían regido y que, por tanto, demandan una profunda revisión de las mismas. Exigen nuevas máximas y nuevos guías. Advierte también que se halla inmerso en un estado en el que los no instruidos han perdido la fe en los instruidos y en el cual no hay opiniones fijas ni convicciones fuertes.

A primera vista, pues, Mill retrata una sociedad en la que parece respirarse un aire de cambio que se acompaña necesariamente de una fuerte dosis de movimiento tanto en el ámbito político como intelectual. De todos modos, se muestra dubitativo respecto del horizonte hacia el que ésta se encamina. Le preocupa particularmente que en este aparente estado de "anarquía intelectual", los hombres tan sólo se "inclinen" hacia las nuevas ideas en lugar de abrazarlas y penetrarlas. Constata que son muy pocos los que profesan una gran confianza en sus propias convicciones, mientras que la mayoría cambia de ideas "con cada soplo de viento" 18 . Observa a su alrededor una sociedad que se ha librado de los viejos prejuicios, pero que se encuentra herida de escepticismo y aún no ha logrado adoptar nuevas ideas. Por lo demás - hace constar en otro artículo- el tipo de conocimiento que se ha ido esparciendo entre la multitud gracias al avance de la civilización es extensivo pero superficial. Es "un tipo de instrucción microscópica más que comprensiva, que lleva a ver unas pocas cosas con fuerza y del resto, nada", un tipo de conocimiento que se convierte, al final de cuentas, en "el padre de la estrechez mental y el fanatismo"19. Intuye, así, la posibilidad de que los individuos se internen por caminos de mediocridad y falta de sistematicidad en el que terminen "esclavizándose", no ya a las antiguas autoridades, sino a las personas que se encuentran más cerca suyo, aquellas que, por ejemplo, muestren mayor facilidad para forzar continuamente

${ }^{17}$ Mill también describe a su época como "transitoria" en la reseña del primer volumen de la Democracia en América ( $C W$, Vol. XVIII, p. 54) y en una carta dirigida a Robert B. Fox el 19-2-1842 ( $C W$, Vol. XIV, p. 564).

18 "The Spirit of the Age. I" ( $C W$, Vol XX, p. 233). Enseguida agrega: "Antes de elogiar a un hombre o a una generación por haberse quitado los prejuicios, quiero saber por qué los han substituido". La misma advertencia se reitera en "The English National Character", en donde escribe: "Nos estamos quitando de encima rápidamente nuestras viejas convicciones pero no nos estamos formando unas nuevas" ( $C W$, Vol. XXIII, p. 272).

${ }^{19}$ Cfr. "Notes on the Newspapers" (CW, Vol. VI, p. 154). 
su atención hacia las conclusiones favorables a las que ellos mismos habrían querido arribar.

En definitiva, si la sociedad se encuentra atravesando un período de transición, que tarde o temprano será traspuesto, lo que interesa a Mill es —ante todo- vislumbrar la dirección y los nuevos rasgos que ésta habrá de adoptar en la siguiente etapa orgánica. En este orden, comienza a preguntarse si la movilidad y el cambio con los que varios identifican a su época son acaso rasgos que habrán de perdurar o, por el contrario, si la sociedad moderna se orienta en realidad hacia un tipo de inmovilidad más profunda, y el movimiento que se observa resulta tan sólo uno de tipo epidérmico.

\section{El estancamiento como un mal propio de las sociedades "civilizadas" o "modernas"}

Mill nunca abandonó por completo el optimismo respecto del futuro de la sociedad moderna ${ }^{20}$. No obstante, la experiencia política francesa (que tan de cerca siguió) y el contacto con el liberalismo continental lo fueron llevando a identificar al estancamiento como uno de los rasgos distintivos de aquellas sociedades que habían alcanzado un determinado estadio de civilización ${ }^{21}$. De hecho, en abril de 1836 apa-

${ }^{20}$ Hasta el final de sus días el inglés reiteró su convicción respecto de que los peligros que acechaban a sus contemporáneos eran removibles con grandes esfuerzos y con el concurso de varias generaciones (Cfr. Utilitarism, $C W$, Vol. X, pp. 216-217). Como confiesa al americano Ch. Cummings a comienzos de 1863, pocas personas miraban la carrera futura de la Humanidad con mayores esperanzas que él (Cfr. $C W$, Vol. XV, p. 843).

${ }^{21}$ En el libro VI de $A$ System of Logic Mill define el "estado de la sociedad" o "estadio de civilización" de la siguiente manera: "Se llama así al estado, en un mismo momento, de todos los hechos o fenómenos sociales más importantes. Tales son el grado de instrucción, de cultura intelectual o moral alcanzada por la comunidad o por cada una de las clases que ésta comprende; el estado de la industria, de la riqueza y de su distribución; las ocupaciones ordinarias del grupo social, su división en diferentes clases y las relaciones de estas clases entre sí; las creencias comunes sobre las cuestiones que interesan más a la Humanidad y la fuerza de convicción con que se adhiere a ellas; sus gustos y el carácter como el nivel de su desarrollo estético; la forma de gobierno adoptada, las principales leyes o costumbres, etc. La condición de estos diferentes factores y de un gran número de otros, cuya idea acudirá fácilmente al espíritu; he aquí lo que constituye el estado de sociedad o el estado de civilización de una época dada" ( $C W$, Vol. VIII, pp. 911-912). 
rece publicado su artículo "Civilization: Signs of the Times", en el que afirma sin rodeos que la civilización debe ser considerada como un bien y como "la causa de mucho bien", antes que como un fenómeno incompatible con él. Pero, acto seguido, admite que existe "otro bien, un bien más alto, que la civilización [entendida en sentido restringido] no asegura, y parte del cual tiene una tendencia a impedir"22. En algunos ámbitos, precisa, su época se revela "estacionaria" y hasta "retrógrada".

¿Por qué cree Mill que el crecimiento de la civilización conduce al estancamiento? ¿Cómo explica que una sociedad civilizada pierda vigor y dinamismo y proyecte, en cambio, una imagen de inercia y aparente tranquilidad? Parte de la respuesta a este interrogante reside en un fenómeno completamente nuevo al que califica como "la consecuencia más extraordinaria" que ha reportado el avance natural de la civilización. Se trata del crecimiento de las masas, de su peso e importancia, y el empequeñecimiento y la insignificancia que experimentan los individuos cuando se comparan con ella ${ }^{23}$. En efecto, la pérdida de visibilidad de los individuos tomados aisladamente, su debilidad y desorientación, es lo que explica la disposición que éstos manifiestan hacia el retraimiento y la indiferencia que proyectan hacia la cosa pública. Para Mill resulta muy difícil esperar que una persona pueda desarrollar un sentimiento vivo y verdadero de patriotismo cuando no se encuentra envuelta de ninguna forma en la responsabilidad de los asuntos públicos, y no puede esperar ejercer "más que una mínima influencia sobre ellos" 24 . Apoyado en las intuiciones apuntadas por Tocqueville, sostiene que cuando los hombres abdican de su condición de ciudadanos y se repliegan en su esfera individual, la vida política se paraliza. En un escenario de individualismo y desafección pública, no

22 "Civilization" ( $C W$, Vol. XVIII, p. 119). Probablemente tributaria de la distinción realizada por Guizot en sus lecciones sobre la Histoire de la Civilisation en Europe, Mill sostiene que el término "civilización" puede entenderse de dos maneras. En un sentido amplio, un país civilizado es aquel que ha alcanzado un alto desarrollo de las características más eminentes del hombre y la sociedad, que "ha avanzado en el camino hacia la perfección" y se manifiesta más "feliz, noble y sabio". En cambio, en un sentido restringido, la civilización se refiere específicamente a "un tipo de progreso que diferencia a una nación rica y poderosa, de las salvajes o bárbaras". Cfr. Mill, "Guizot's Lectures on European Civilization", London Review (enero 1836), (CW, Vol. XX, pp. 367-393).

23 "Civilization", $C W$, Vol. XVIII, p. 121. Cfr. también On Liberty $(C W$, Vol. XVIII, p. 268).

24 “De Tocqueville on America. II” ( $C W$, Vol. XXIII, p. 182). 
hay manera de hacer oír las diferentes necesidades e intereses que recorren la sociedad; los canales de comunicación con el gobierno pierden eficacia y se corrompen, y tampoco es posible que el pueblo ejerza el control último de la soberanía ${ }^{25}$. De esta suerte, la política queda en manos de un grupo restringido, con escasos incentivos para modificar el curso de acción.

El avance de la civilización también contribuye a moldear un cierto tipo de carácter en los individuos que integran las sociedades modernas. Y este carácter, que Mill denomina "espíritu comercial", favorece la tendencia hacia el estancamiento intelectual y moral. Entre sus rasgos más salientes se destacan, en primer lugar, la relajación de la energía individual, o más exactamente, "la concentración de la misma en la reducida esfera de la consecución de los intereses materiales" 26 . A medida que la civilización avanza, los hombres pasan a depender cada vez más de los arreglos generales de la sociedad en lugar de sus propios esfuerzos a la hora de satisfacer sus necesidades más inmediatas (léase, su seguridad personal, la protección de su familia, su propiedad o su libertad). En segundo lugar, y en estrecha relación con el punto anterior, la "universalización" de una pasión propia de las clases medias que, aparentemente, amenaza con expandirse sobre el resto de la sociedad: el amor al dinero y al bienestar material ${ }^{27}$. Ocupados únicamente en hacer fortuna, los hombres modernos se vuelven incapaces de apreciar los placeres intelectuales y marchan hacia una mediocridad

25 Cfr. "Pledges", Examiner (1-7-1832), (CW, Vol. XXIII, p. 489) y "Rationale of Representation", London Review (julio 1835), (CW, Vol. XVIII, p. 24). Aunque no es posible extender aquí el análisis sobre este punto, cabe señalar que si bien Mill utiliza el neologismo "individualismo" en contadas ocasiones y con una significación que difiere de la que le asigna Tocqueville, lo cierto es que los rasgos con los cuales ambos describen este fenómeno son llamativamente similares. Cfr. Pollitzer, M. "Individuos Perdidos en la Multitud: Mill y Tocqueville sobre la Sociedad Democrática”, 2012, pp. 5-34.

26 "Civilization" ( $C W$, Vol. XVIII, p. 129) y On Liberty $(C W$, Vol. XVIII, p. 272).

27 “Civilization” (CW, Vol. XVIII, p. 130). En el capítulo III de Considerations on Representative Government Mill concede que — salvo unos pocos- la mayoría de los hombres, en lugar de interesarse por la especulación en sí misma, dirige sus sentimientos y su inteligencia hacia los intereses materiales. $\mathrm{Y}$ en "The English National Character" precisa que "a menudo ellos son inconscientes (...) de esto; siguen un impulso ciego y mecánico, que hace del dinero y de la reputación de tener dinero, el fin inmediato de sus acciones, sin que sepan que esto es así, y menos aún, por qué lo es" ( $C W$, Vol. XXIII, p. 721). 
"insípida"28. Al mismo tiempo, esta pasión predominante nubla sus intereses y los lleva a perder de vista el lazo que une a su bien particular con la prosperidad general. En tercer lugar, la pérdida del espíritu heroico y el rechazo frente a cualquier espectáculo que implique dolor y sufrimiento ${ }^{29}$. En una sociedad civilizada casi todas las ocasiones en las que es preciso provocar dolor son delegadas por un consentimiento común a un grupo de agentes reducido: el juez, el soldado, el cirujano, el carnicero o el verdugo. De hecho, el refinamiento del que muchos se glorían consiste justamente en evitar la presencia no sólo del sufrimiento sino de cualquier tipo de idea desagradable u ofensiva. Esto es posible gracias a que se han ido perfeccionando los arreglos mecánicos que otrora eran impracticables. En cualquier caso, sin desconocer los beneficios que esta realidad reporta, lo cierto es que también oculta un costado poco favorable. Si "el heroísmo consiste esencialmente en estar preparado para hacer o sufrir, pero especialmente para hacer aquello que es doloroso o desagradable siempre que se tenga como meta un objetivo valioso" 30 , quien no tiene ocasión de aprender dicha capacidad, nunca la desarrollará. Como sugiere I. Hampsher-Monk, Mill se hace eco de la exposición que Maquiavelo había realizado sobre los efectos de la necessità en la virtù cuando explica que la seguridad que proporciona la sociedad civilizada elimina lo que en un estado bárbaro constituía el incentivo necesario para el ejercicio de la energía y la astucia individual $^{31}$. En efecto, lo que Mill observa a su alrededor es una

28 "Notes on the Newspapers" ( $C W$, Vol. VI, p. 173). En verdad, para Mill, ellos no eligen "voluntariamente" los placeres inferiores por sobre los superiores, sino que se hallan "incapacitados" para elegir los últimos. Como explica en Utilitarism, el problema está en que faltan las condiciones necesarias para que el gusto por los bienes superiores pueda desarrollarse y los únicos placeres a los que la mayoría tiene acceso y de los que puede gozar son los placeres inferiores. (Cfr. Utilitarism, $C W$, Vol. X, p. 213). Entre los factores que alientan esta escala de preferencias se destaca particularmente el apego que estos hombres profesan al tiempo presente.

${ }^{29}$ Cfr. "Civilization" ( $C W$, Vol. XVIII, p. 130). En "The Negro Question", Fraser's Magazine (enero de 1850), Mill reitera que "la abolición de la inflexión de dolor es (...) la ocupación de esta edad" ( $C W$, Vol. XXI, p. 374).

30 “Civilization" (CW, Vol. XVIII, p. 131).

31 I. Hampsher-Monk, Historia del Pensamiento Político Moderno, 1996, p. 402. Esta misma idea se reitera en el cap. XI del libro V de sus The Principles of Political Economy. Allí comenta que resulta muy difícil que los hombres mantengan un espíritu activo y vigoroso cuando el avance de la civilización y la seguridad ha removido una tras otra las dificultades, las penas y los peligros frente a los cuales antiguamente éstos no tenían más recurso que oponer su propia fuerza, habilidad y coraje (Cfr. $C W$, Vol. III, p. 943). 
"moral afeminada"32 y una preocupante incapacidad para emprender y sostener cualquier tipo de lucha.

Todos estos elementos informan el espíritu prevaleciente entre las clases medias, es decir, entre la mayoría de los hombres en las sociedades civilizadas. Semejante carácter, débil, encogido y apático, que no reconoce el atractivo y la riqueza contenida en los placeres de orden espiritual, conduce a un empequeñecimiento de la vida moral e intelectual de quienes lo encarnan. Esta dimensión de su existencia queda relegada, abandonada. No es objeto de cultivo, sino que permanece en un delicado estado de inmovilidad, un estado que, claro está, no es precisamente inocuo. Por encima de cada uno de estos hombres, cuya vida moral e intelectual se encuentra en cierta medida "indefensa", se alza una masa que crece día a día y amenaza con "dominar al mundo"33.

${ }^{32}$ No deja de sorprender la utilización de este tipo de expresiones de boca de quien fuera uno de los primeros pensadores liberales en discutir los prejuicios asociados a las mujeres y al rol que les cabía dentro de la sociedad. Lo cierto es que, cuando Mill se refiere al costo o los efectos perjudiciales que ha traído el avance de la civilización, se hace eco de la terminología en boga y describe la contextura moral prevaleciente como "afeminada" ("Civilization", $C W$, Vol. XVIII, p. 131) o bien, alude al "encogimiento/acobardamiento afeminado ante la mera sombra de dolor" ("Coleridge", $C W$, Vol. X, p. 123). Este sesgo peyorativo con el que aparece asociado "lo femenino" también se encuentra, a modo de ejemplo, en la segunda reseña que realiza sobre la obra de Tocqueville, cuando habla del establecimiento de una "sensualidad regulada" a la que caracteriza como "el padre del afeminamiento antes que del libertinaje" ("De Tocqueville on Democracy in America. II", $C W$, Vol. XVIII, p. 187); en algunos de sus artículos periodísticos en los que menciona que las clases trabajadoras no se parecen a sus "irritados y afeminados superiores", sino que están acostumbradas al sufrimiento y entrenadas en la fortaleza ("French News", $C W$, Vol. XXIII, p. 371) y en intervenciones parlamentarias en las que denuncia un "debilitamiento, un afeminamiento, del espíritu general del país" ("Capital $\mathrm{Pu}$ nishment”, $C W$, Vol. XXVIII, p. 269). En estos casos, la adjetivación escogida no se halla acompañada por ningún tipo de comentario aclaratorio que relativice o desmienta aquello que sus palabras indican implícitamente, es decir, la existencia de una asociación estrecha entre lo femenino y la debilidad, el encogimiento o la mediocridad ¿Acaso su silencio sobre esta materia revela su conformidad con la misma, y, en ese caso, una posible inconsistencia con los argumentos presentados, por ejemplo, en The Subjection of Women? ¿O se trata simplemente de una suerte de "concesión literaria", de un "lugar común" que Mill adopta de sin mayor deliberación? Se trata de una pregunta para la cual no tengo aún una respuesta pero que abre una línea de investigación por demás interesante. Agradezco a uno de los revisores por haber llamado mi atención sobre este punto.

${ }^{33}$ On Liberty (CW, Vol. XVIII, p. 268). 
Al crecimiento de la masa y la opacidad de individuos impregnados de este "espíritu comercial", Mill agrega dos factores más que favorecen el establecimiento de un escenario de estancamiento en las sociedades modernas. Pero la particularidad de estos factores (el predominio del miedo y la confusión entre el deseo de libertad y el ansia de poder) radica en que en lugar de estar estrictamente vinculados con el avance de la civilización sin más, se manifiestan, en todo caso, como rasgos salientes de un determinado carácter nacional, el del pueblo francés. Ello nos obliga a sumar una nueva perspectiva de análisis a la comprensión de este peligro.

\section{El estancamiento como horizonte de determinados caracteres nacionales}

En el siglo XIX, Francia e Inglaterra se encontraban en el mismo estadio de la civilización, pero sus caracteres nacionales eran ciertamente distintos. Mill da cuenta de las diferencias que separan a ambas naciones en repetidas oportunidades a lo largo de su vida ${ }^{34}$. Según aduce G. Varouxakis, el desencanto con la "jeune France" y los acontecimientos inmediatos a la revolución de 1848, hicieron que el inglés fuera corrigiendo la sobreestimación que inicialmente había adjudicado

${ }^{34}$ Desde muy joven, sobre todo a partir de su primer viaje a Francia, el inglés percibió el contraste entre "la manera de ser" de sus connacionales y la de sus vecinos continentales. En la década del 30 el tema aparece en varios de sus artículos periodísticos, de los que cabe destacar al menos dos: "Comparison of the Tendencies of the French and English Intellect" ( $\mathrm{CW}$, Vol. XXIII), publicado en el Monthly Repository en noviembre de 1833 (originalmente, había sido publicado en el periódico saint-simoniano Le Globe), y "The English National Character" ( $C W$, Vol. XXIII), publicado también en el Monthly Repository en junio de 1834 (pero originalmente pensado como una carta dirigida a A. Carrell y a su periódico Le National). Asimismo, se lo encuentra presente en la correspondencia mantenida con los franceses G. d'Eichthal (Cfr. especialmente las cartas fechadas los días 15-5-1829 y el 9-2-1830), A. Comte (Cfr. cartas fechadas los días 22-3-1842 y 6-5-1842) y Tocqueville (Cfr. la carta con fecha del 30-12-1840), en la mayoría de las reseñas de las obras de autoría francesa (como por ejemplo "Mignet's French Revolution" [ $C W, \mathrm{Vol} . \mathrm{XX}]$, "Modern French Historical Works" [CW, Vol. XX], las dos reseñas realizadas sobre las obras de Guizot y "Armand Carrell” [CW, Vol. XX]) y, más adelante, en Considerations on Representative Government y Subjection of Women. Cfr. también "The Peerage Question in France", Examiner (4-9-1831), (CW, Vol. XXIII) “The Spirit of the Age" [1831] (CW, Vol. XXII) y "Sedgwick's Discourse", London Review (abril de 1835). (CW, Vol. X). 
al cambio generacional y comenzara a otorgar mayor importancia a los factores "más duraderos" del carácter nacional a la hora de estimar las probabilidades para el establecimiento de un régimen libre ${ }^{35}$. A modo de ejemplo, en sus Principles of Political Economy, sostiene que "en algunos países el deseo de los hombres es no ser tiranizados, mientras que en otros consiste meramente en tener igualdad de oportunidades para tiranizar"36. Una hipótesis que reitera luego en el capítulo III de Considerations on Representative Government, cuando analiza los obstáculos que dificultan el disfrute de los beneficios del gobierno representativo: hay entre los hombres dos tendencias que operan en direcciones contrarias - aclara - , una es el deseo de ejercer poder sobre los otros y la otra es el rechazo a que el poder sea ejercido sobre uno mismo. Así, "hay naciones en las que la pasión por gobernar a otros es más fuerte que el deseo de la independencia personal, [de modo que] por la mera sombra de una se encuentran listos para sacrificar la totalidad de la otra" 37 . Grafica su argumento con el caso del soldado que es capaz de abdicar su libertad de acción personal en las manos de su general siempre que el ejército sea triunfante y victorioso, y que se gloría de ser parte de una multitud conquistadora, a pesar de que la idea que tiene sobre el peso que a él mismo le cabe en el dominio ejercido sobre los conquistados, sea una mera ilusión.

Para Mill, este tipo de hombres no está interesado en un gobierno limitado en sus atribuciones y su poder. Por el contrario, ve en el crecimiento del estado una oportunidad para obtener cargos públicos (fenómeno que describe como "place-hunting"). En realidad lo que prefiere es la posibilidad, no obstante lo lejana e improbable que ella sea, de ejercer el poder sobre sus conciudadanos a la certeza de que no se está ejerciendo un poder innecesario sobre sí mismo. De esta forma, aprecia la igualdad pero no la libertad. O, como indica en otra ocasión,

35 Cfr. G. Varouxakis, "National Character in J. S. Mill's Thought", 1998 , p. 380.

${ }^{36}$ The Principles of Political Economy ( $C W$, Vol. III, p. 944).

37 Considerations on Representative Government ( $C W$, Vol. XIX, p. 420). Detrás del análisis que Mill ofrece en este punto se pueden percibir los ecos rousseaunianos que alertaban, hacia 1755, acerca de los "ciudadanos [que] no se dejan oprimir sino arrastrados por una ciega ambición, y [que] al mirar más por debajo de ellos que por encima, la dominación les parece más querida que la independencia y consienten en llevar cadenas para poder ponerlas a su vez" (Rousseau, Discurso Acerca del Origen de la Desigualdad entre los Hombres [1755], 2001, p. 133). 
confunde el amor a la libertad con el amor al poder, lo que constituye no sólo un "error psicológico", sino también "la peor lección moral posible". A diferencia del primero, el apetito por el poder es egoísta, en la medida en que el poder de uno implica la ausencia del mismo en el otro. Es la pasión más peligrosa y dañina de la naturaleza humana puesto que "todo poder sobre otros (a excepción de aquel que da la influencia moral e intelectual) implica coerción y compulsión". Incluso en casos indispensables, éste resulta una "trampa" y, en la mayoría, "una maldición, tanto para los que lo ejercen como para los que lo padecen"38.

La descripción del carácter prevaleciente en la nación francesa se ofrece como contrapunto de aquel que observa entre sus connacionales. Los ingleses - declara - recelan cualquier tentativa en la que un poder excesivo, que no cuente con la sanción de la costumbre o de su propia opinión sobre lo que es correcto, pueda ser ejercido sobre ellos. Les importa poco ejercer poder sobre otros, no tienen pasión por gobernar y (aunque pueda resultar difícil de entender para los extranjeros) prefieren que de ello se ocupen aquellos que no buscan el poder directamente sino que lo ejercen más bien como consecuencia de su posición social. No les gusta la lucha por los cargos públicos y miran con gran aversión la multiplicación de los mismos, fenómeno muy popular en las naciones del continente. Es más, entre ellos, la intervención del estado es vista como "un remedio extremo" que debe actuar únicamente en las grandes ocasiones, en los momentos difíciles o en aquellas cuestiones tan vitales que no puedan ser confiadas a manos menos responsables ${ }^{39}$.

Un segundo rasgo que llama su atención y lo preocupa es el imperio alcanzado por el miedo en la Francia de Luis Felipe. "Nada hay que temer más que el hábito de temer" 40 — anota en una de sus primeras contribuciones periodísticas. El miedo paraliza, nubla la mente y conduce a la "oposición ignorante al cambio"41. Ahora bien, ¿sobre qué se proyecta este miedo que él considera tan extendido? Claramente sobre la posibilidad de nuevas revoluciones que interrumpan el tímido orden recuperado. En su opinión, no hay ningún otro país en el mundo en el que los peligros de una convulsión popular sean más temidos que en

38 "Centralisation" ( $C W$, Vol. XIX, p. 610).

39 “Centralisation" ( $C W$, Vol. XIX, p. 609).

40 "Law of Libel and the Liberty of the Press", Westminster Review (abril 1825), (CW, Vol. XXI, p. 12).

${ }^{41}$ Chapters on Socialism (CW, Vol. V, p. 708). 
Francia. En uno de sus artículos publicado bajo el título de "Prospects of France" Mill reconoce que, al haber experimentado en carne propia lo que significa una verdadera revolución, los franceses "cierran sus casas a cada llamado de la puerta, y escuchan el ruido de la anarquía y la devastación en cada brisa". En contrapartida, los ingleses no están acostumbrados a pensar la revolución bajo la categoría de "fenómeno posible", "sólo [su] razón y no [su] imaginación cree en esa posibilidad". Sus vidas transcurren con la acostumbrada regularidad al tiempo que proclaman estar “al filo del abismo". Es más, si realmente se llegara a producir una revolución en Gran Bretaña, insinúa, sus consecuencias serían mucho más catastróficas que las que podrían llegar a afectar a Francia $^{42}$.

Es probable, entonces, que la ausencia de una auténtica experiencia revolucionaria en el suelo inglés sea lo que le permite a Mill saludar a las ideas socialistas que tanto asustan a los sectores más acomodados en Gran Bretaña y en Francia, como aquellas capaces de sacudir el statu quo y, lejos de paralizar a la sociedad, forzar en ella la discusión y la revisión de las ideas establecidas. Una sociedad estancada es una sociedad que descansa sobre una falsa seguridad, en virtud de la cual se minimiza o silencia toda aquella opinión que pueda llegar a amenazarla. De hecho, junto a la indiferencia política, el miedo es uno de los obstáculos que impiden (según Mill) que la nación vecina alcance de manera definitiva el gobierno representativo ${ }^{43}$.

En síntesis, para Mill el problema del estancamiento se enraíza con el avance de la civilización y aplica particularmente a Francia, aunque también se observa en Inglaterra. La progresiva desilusión generada por el régimen burgués liderado por Luis Felipe de Orléans, sumado al ascenso de Luis Napoleón y el fracaso de la II República, llevaron a Mill a admitir que - efectivamente- Francia se hallaba presa del estancamiento político, mal que, por otro lado, permanecía aún tan sólo como una amenaza para los ingleses. Entre ellos, el estancamiento anidaba principalmente en el ámbito moral e intelectual ${ }^{44}$. Al parecer,

\footnotetext{
42 "Prospects of France.VIII", Examiner (10-4-1831), (CW, Vol. XXII, p. 297).

43 "Vindication of the French Revolution of February 1848", Westminster Review (abril 1849), (CW, Vol. XX, p. 332).

${ }^{44}$ Cfr. la carta dirigida a P. Villari el 9-3-1858 (CW, Vol. XV, p. 550) у a otra dirigida a T. Comperz, el 5-10-1857 (CW, Vol. XV, p. 539).
} 
entonces, este último tipo de estancamiento podía llegar a darse sin el concurso del primero, aunque (como admite en una carta hacia 1850) también en Inglaterra "[se había] llegado (...) a un período en el que el progreso, inclusive el de tipo político, [estaba] parado a causa del bajo estado moral e intelectual de todas las clases" ${ }^{25}$. En cualquier caso, la relación inversa es poco probable. Implícitamente, su diagnóstico indica que el estancamiento político tiende a acompañar de manera natural al estancamiento moral e intelectual de toda la sociedad y, si no se lo combate abiertamente, termina conduciendo directamente hacia él.

\section{El estancamiento como peligro recurrente}

El fenómeno del estancamiento tal como lo visualizó Mill admite un último matiz interpretativo. En el fondo, para él, esta problemática se encuentra estrechamente vinculada con la perspectiva de una sociedad cada vez más uniforme en la que una sola clase, un solo tipo de ideas, gustos e intereses, se impongan sobre el resto de manera hegemónica, eliminando o confinando a la insignificancia cualquier tipo de contrapeso. "Una comunidad homogénea es naturalmente una comunidad estacionaria"46 — sentencia hacia 1840_. De hecho, ésta fue una de las lecciones más valiosas que Mill supo incorporar tras la lectura de las obras de F. Guizot. Según el historiador francés, la clave del progreso y la libertad que distinguían a la civilización europea de los estados orientales radicaba, precisamente, en el hecho de que la primera siempre había estado signada por la existencia de fuerzas, principios y sistemas diversos que luchaban sin cesar, se limitaban entre sí y eran incapaces de excluirse mutuamente. Este sano antagonismo era la razón de su dinamismo y vitalidad ${ }^{47}$.

${ }^{45}$ Cfr. carta a Herford, fechada el $22-1-1850$ ( $C W$, Vol. XIV, p. 45).

46 "De Tocqueville on Democracy in America. II" ( $C W$, Vol. XVIII, p. 196).

${ }^{47}$ Mill se vale de la imagen del estancamiento "chino" en varias oportunidades. Aparece en primer lugar en el ensayo sobre "Bentham", como ejemplo de lo que puede ocurrir cuando en una sociedad no se mantiene vivo el antagonismo y no existe un "centro de resistencia alrededor del cual todos los elementos morales y sociales que son vistos con desaprobación por el poder gobernante puedan agruparse", y encontrar refugio frente a los intentos por parte del poder de turno para borrarlos de la existencia. En segundo lugar, siguiendo un orden cronológico, la alusión a China también aparece en la reseña que publica 
Siguiendo esta lógica argumental, Mill recuerda que todas las naciones que fueron progresistas conocieron "una oposición organizada frente al poder gobernante, cualquiera fuera la forma que este poder tuviera: plebeyos contra patricios, el clero contra los reyes, libre-pensadores contra el clero, reyes contra barones o Comunes contra el rey y la aristocracia" 48 . En la segunda reseña que publica sobre las obras de Guizot llega a afirmar, incluso, que el antagonismo es "la única condición bajo la cual la estabilidad y el progreso pueden ser reconciliados de manera permanente" 49 . En efecto, cuando cesa el conflicto entre el poder más fuerte de una comunidad y su poder rival, es decir, "cuan-

sobre el segundo volumen de la Democracia en América (véase nota $\mathrm{N}^{\circ}$ 6). A los pocos años es evocada en una carta dirigida a A. Comte en febrero de 1842 ( $C W$, Vol. XIII, pp. 501-504) y en la segunda reseña de las obras de Guizot en 1845 ( $C W$, Vol. XX, p. 270). En ambos casos, el país asiático ejemplifica los riesgos que implica el hecho de que una sociedad sea gobernada por una elite intelectual que ejerce un poder de tutelaje exclusivo sobre todos los aspectos de la vida. Es la prueba viviente de que la apuesta por el poder espiritual, en la versión saint-simoniana que lo había seducido en un principio, lejos de alcanzar los resultados esperados, abrigaba un costado muy peligroso. La clase educada en China se había convertido en lo que aquí llama una "pedantocracia". Otorgar a cualquier grupo una autoridad exclusiva y sin control, permitir la existencia de una preponderancia absoluta incluso del "elemento más saludable" de la sociedad como puede ser el sector letrado y culto, resulta siempre una alternativa riesgosa.

China también le sirve a Mill como espejo de una sociedad uniforme. En el capítulo III de su famoso ensayo On Liberty afirma que los chinos han alcanzado con gran éxito aquella meta a la que los filántropos europeos parecen apuntar: "el hacer a todos los hombres iguales". Sostiene que "el moderno régimen de la opinión pública es, en una forma no organizada, lo que los sistemas político y educativo en China son en una forma organizada" ( $C W$, Vol. XVIII, p. 274). Compara la acción de la opinión pública sobre el carácter general de los hombres con la imagen del pie de una mujer china, en el que se mantiene comprimida cualquier parte de la naturaleza humana que sobresalga de manera prominente.

Por último, cabe señalar también que en Considerations on Representative Government, Mill asegura que si se pierden los frutos de la libertad, que son la energía, el patriotismo y la apertura mental, Inglaterra puede degenerar en pocas generaciones en un estado oriental ( $C W$, Vol. XIX, p. 401).

48 "Bentham", London and Westminster Review (agosto 1838), (CW, Vol. X, p. 108).

49 "Guizot's Essays and Lectures on History", $C W$, Vol. XX, p. 269. Mill utiliza la expresión "antagonismo indispensable" en una carta dirigida a A. Comte el 25-2-1842 (Cfr. CW, Vol. XIII, p. 503) y en "Guizot's Essays and Lectures on History" habla de un "antagonismo sistemático" (Cfr. CW, Vol. XX, p. 270). 
do una de las partes logra imponer su posición de manera completa y anula la posibilidad de la contienda, llega el estancamiento y luego la decadencia" 50 . En Considerations on Representative Government sugiere, además, que la existencia de influencias conflictivas es de capital importancia "en todos los asuntos humanos", ya que la prosecución exclusiva de un único objeto, en lugar de generar el exceso en aquel que se busca y el defecto en el que se ignora, "conduce al deterioro y la pérdida de aquello que únicamente se buscaba" 51 .

Como puede observarse, para Mill la posibilidad del estancamiento no es un mal privativo del pasado o, en todo caso, de los estados orientales. Constituye, más bien, una amenaza omnipresente, un peligro recurrente que se materializa toda vez que el antagonismo da paso a la preponderancia de uno de los componentes asociados en la contienda. En las sociedades civilizadas o democráticas, como la americana, el elemento predominante tiende a ser la clase media. De ahí que para nuestro autor, América no se encuentra exenta del peligro que aquí se denuncia.

Ahora bien, en rigor de verdad, el poder que confiere el número (aquel del que gozan las clases medias), no es —en sí mismo- el centro del problema. Como precisa en su reseña al segundo volumen de la Democracia en América, "el gran peligro no es la preponderancia de la clase democrática, sino la de cualquier clase" $" 52$. Un punto que reitera a los pocos años: "Lo que es formidable no es el ascenso descontrolado del poder popular, sino de cualquier poder". ¿Por qué? Porque ni bien un poder reina sin control, ni bien "se encuentra exento de la necesidad de estar en lo cierto porque es capaz de hacer que su voluntad prevalezca sin tener que atravesar por una lucha previa", sus influencias se convierten en perniciosas. En conclusión, "para lograr que el ascenso del poder popular sea seguro, es necesario poner a su lado los correctivos y contrapesos que posean aquellas cualidades opuestas a las de sus defectos característicos"53. O bien, garantizar la existencia de un "centro de resistencia alrededor del cual todos los elementos morales y sociales que son vistos con desaprobación por el poder gobernante puedan agruparse, y, bajo su protección, encontrar refugio frente a los intentos

${ }^{50}$ Considerations on Representative Government ( $C W$, Vol. XIX, p. 458).

${ }^{51}$ Considerations on Representative Government ( $C W$, Vol. XIX, p. 439).

52 "De Tocqueville on Democracy in America. II" (CW, Vol. XIX, p. 196).

53 "Duveryrier's Political Views of French Affairs", Edinburgh Review (abril 1846), (CW, Vol. XX, p. 306). 
de dicho poder para borrarlos de la existencia"54. Sobre esta senda se encolumnan sus propuestas concretas: en el nivel institucional, mejorar los mecanismos de representación a fin de garantizar un lugar en el gobierno a los sectores que pudieran oficiar de contrapunto frente a la mayoría, alentar la descentralización administrativa y propiciar la participación ciudadana en los gobiernos municipales y en las asociaciones voluntarias. En lo que respecta a las herramientas a través de las cuales entendía que podía mantenerse viva la heterogeneidad social —amén de los dispositivos institucionales - se destacan su alegato a favor de la necesaria coexistencia de diversas clases sociales (clase media urbana, clase agrícola, clase ociosa y clase letrada ${ }^{55}$ ); la esperanza depositada en la renovación y la extensión de la educación; su férrea defensa de la libertad de pensamiento y discusión, materializada en la libertad de prensa, e inclusive, su llamado a la genialidad y la excentricidad ${ }^{56}$.

54 "Bentham", $C W$, Vol. X, p. 108. La misma idea se reitera en The Principles of Political Economy ( $C W$, Vol. III p. 940), en On Liberty ( $C W$, Vol. XVIII, p. 323) y en Considerations on Representative Government (CW, Vol. XIX, p. 459). ${ }^{55} \mathrm{Cfr}$. "De Tocqueville On Democracy in America. II" ( $C W$, Vol. XVIII, p. 198).

${ }^{56}$ En una de sus reseñas sobre la Historia de Grecia de Grote, Mill indica que "una sociedad que mira con celos y desconfianza a la gente original, que impone su común nivel de opinión, sentimiento y conducta a todos sus miembros individuales puede tener la satisfacción de pensar que es muy moral y respetable, pero no puede tener genios" ( $C W$, Vol. XI, pp. 320-321). Siguiendo a Von Humboldt, cree que la individualidad supone originalidad, y ésta "la capacidad de extraer el conocimiento de una verdad general de nuestro propio conocimiento, [ya sea] a través de la simple observación, a través de ese tipo de auto-observación que algunos llaman imaginación, o a través de un proceso de análisis e inducción más complicado" ("On Genius", Monthly Repository octubre 1832, en CW, Vol. I, p. 332). Conocer una verdad significa, de alguna manera, descubrirla, hacerla propia. Pero ello se dificulta en una atmósfera de creciente uniformidad en la que se impone la tiranía de la opinión y en el que los hombres carecen de heroísmo y se hallan incapacitados para "afrontar cualquier trabajo, (...), aguantar el ridículo, (...) desafiar las malas lenguas, (...) o decir con intrepidez algo desagradable a alguien a quien están acostumbrados a ver" ("Civilization", $C W$, Vol, XVIII, p. 131). Es en este marco que Mill alienta a los hombres a que desafíen los mandatos sociales. Incluso llega a afirmar que "en orden a romper con esta tiranía, los hombres deberían ser excéntricos" (On Liberty, CW, Vol. XVIII, p. 269). Según M. Hopenhayn, la excentricidad opera, en la reflexión de Mill, precisamente como una "inyección de movimiento" que evita que las aguas se estanquen. La mera existencia del excéntrico interpela al resto, los fuerza a revisar los fundamentos en los que descansan sus propias ideas y sentimientos y, en definitiva, los sacude de la rutina conformista en la que se encuentran sumidos. (Cfr. "La Libertad y la Tentación de la Excentricidad”, Estudios Públicos, № 46, 1992, pp. 95-112) 


\section{Conclusión}

La preocupación por un horizonte de estancamiento recorre gran parte de la obra de John Stuart Mill y se encuentra estrechamente vinculada con las ideas centrales de su reflexión política. Si atendemos a sus palabras, la inmovilidad "a la china" se presenta como un peligro que se encuentra más próximo de lo que muchos imaginan y cuyos efectos pocos logran entrever. Como se dijo, es un mal que se despliega tanto en el ámbito moral e intelectual como en la arena política y se erige como la antesala de nuevas formas de despotismo. Se trata de un fenómeno estrechamente vinculado con la naturaleza misma de las sociedades democráticas o civilizadas, puesto que hunde sus raíces en los cambios que trae aparejados el avance de la civilización: entre otros, el crecimiento de las masas; la debilidad, opacidad y desorientación de los individuos; el creciente individualismo y la desafección pública y la preeminencia del "espíritu comercial" que lleva a que los hombres prioricen su bienestar material, circunscriban sus energías y aspiraciones en empresas poco elevadas, limiten sus ambiciones y vean diluida su esfera de autonomía individual. Es también un escenario particularmente peligroso para determinados pueblos, aquellos en los que imperan el miedo y la resistencia al cambio y en los que la pasión por la igualdad lleva a los hombres a descuidar el amor por la libertad o a confundirlo con el amor al poder. Pero sobre todo, el gran lazarillo, el verdadero asiento del estancamiento es la uniformidad en materia intelectual y la homogeneidad del cuerpo social. En este sentido, el estancamiento se convierte en una amenaza siempre latente que se cierne sobre toda sociedad en la que uno de los poderes o grupos que la integran se impone de manera hegemónica sobre el resto y ahoga el sano antagonismo que la mantenía viva.

Sus reflexiones resuenan aún hoy como una alerta que nos convoca e interpela. Nos recuerdan que las sociedades democráticas que aspiran a engendrar en su seno hombres libres, han de promover estrategias orientadas a mantener y preservar entre ellos un espíritu de sincera apertura hacia la diversidad que no se confunda con una mera tolerancia epidérmica. Han de propiciar, al mismo tiempo, espacios en los cuales tengan cabida el antagonismo y el conflicto que la pluralidad de voces, sentimientos, intereses e ideas necesariamente acarrean. Ya sea en los diseños institucionales, en los distintos grupos y actores sociales como 
"en todos los asuntos humanos"57, la existencia de fuerzas conflictivas — afirma Mill - es saludable, fecunda, denota vitalidad y posibilita el progreso. Ésta es una enseñanza que no deben olvidar los hombres democráticos, quienes suelen enarbolar la bandera de la igualdad con mayor facilidad que la de la libertad. Es también un recordatorio para quienes defienden un discurso que prioriza la búsqueda de consensos desconociendo o minimizando (en ocasiones) los riesgos a los que se abre una sociedad que, agobiada por discordias aparentemente inconducentes, anhela cándidamente un escenario de "pacificación intelectual".

\section{BIBLIOGRAFÍA}

Collini, S. Public Moralists: Political Thought and Intellectual Life in Great Britain 1850-1930. Oxford: Oxford University Press, 1991.

Hampsher-Monk, I., Historia del Pensamiento Político Moderno. Barcelona: Ariel, 1996.

Hopenhayn, M. "La Libertad y la Tentación de la Excentricidad". Estudios Públicos, N 46, 1992, pp. 95-112.

Mill, J. S. Collected Works of John Stuart Mill, ed. J. M. Robson. Toronto: University of Toronto Press. London: Routledge and Kegan Paul, 19631991. 33 vols. [En adelante, $C W$ ]

- A System of Logic Ratiocinative and Inductive, Being a Connected View of the Principles of Evidence and the Methods of Scientific Investigation. En CW, Vol. VII-VIII. 1974.

“Armand Carrell", London and Westminster Review (1837). En CW, Vol. XX: Essays on French History and Historians. 1985.

Autobiography. En CW, Vol. I. Autobiography and Literary Essays. 1981.

"Bentham", London and Westminster Review (agosto 1838). En CW, Vol. X. Essays on Ethics, Religion and Society. 1985.

"Capital Punishment". En $C W$, Vol. XXVIII.

"Centralisation", Edinburgh Review (abril 1862). En $C W$, Vol. XIX. Essays on Politics and Society. Part II. 1977.

Chapters on Socialism. En CW, Vol. V. Essays of Economics and Society. Part II. 1967.

"Civilization", London and Westminster Review (abril 1836). En CW, Vol. XVIII. Essays on Politics and Society. Part I. 1977.

"Coleridge", London and Westminster Review (marzo 1840). En CW, Vol. X. Essays on Ethics, Religion and Society. 1985.

"Comparison of the Tendencies of French and English Intellect", Monthly Repository (noviembre 1833). En CW, Vol. XXIII. Newspaper Writings. August 1831-October 1834. Part II. 1986.

${ }^{57}$ Considerations on Representative Government ( $C W$, Vol. XIX, p. 439). 
Considerations on Representative Government. En CW, Vol. XIX. Essays on Politics and Society. Part II. 1977.

"De Tocqueville on Democracy in America. I", London and Westminster Review (octubre 1835). En CW, Vol. XVIII. Essays on Politics and Society. Part I. 1977.

"De Tocqueville on Democracy in America II", Edinburgh Review (octubre 1840). En CW, Vol. XVIII. Essays on Politics and Society. Part I. 1977.

"Duveryrier's Political Views of French Affairs", Edinburgh Review (abril 1846). En $C W$, Vol. XX

"French and English Journals", Examiner (diciembre 1832). En $C W$, Vol. XXIII. Newspaper Writings. August 1831-October 1834. Part II. 1986.

"French News [15] y [18]", Examiner (1831). En CW, Vol. XXII. Newspaper Writings. December 1822- July 1831. Part I. 1986.

"French News [69] y [73]", Examiner (1832). En CW, Vol. XXIII. Newspaper Writings. August 1831-October 1834. Part II. 1986.

"French News", Examiner (enero 26, 1834). En CW, Vol, XXIII.

"Guizot's Essays and Lectures on History", Edinburgh Review (octubre 1845). En CW, Vol. XX. Essays on French History and Historians. 1985.

“Guizot's Lectures on European Civilization”, Westminster Review (enero 1836). En CW, Vol. XX. Essays on French History and Historians. 1985.

"Law of Libel and Liberty Press", Westminster Review (abril 1825). En CW, Vol. XXI. Essays on Equality, Law and Education. 1984.

"Mignet's French Revolution". Westminster Review (1825). En CW, Vol. XX: Essays on French History and Historians. 1985.

"Modern French Historical Works". Westminster Review (julio 1836). En $C W$, Vol. XX: Essays on French History and Historians, 1985.

"Notes on the Newspapers", Monthly Repository (marzo-agosto 1834). En CW, Vol. VI. Essays on England, Ireland and the Empire. 1982.

“On Genius”, Monthly Repository (octubre 1832). En CW, Vol. I. Autobiography and Literary Essays. 1981.

On Liberty. En CW, Vol. XVIII. Essays on Politics and Society. Part I. 1977.

"Pledges", Examiner (1832). En CW, Vol. XXIII. Newspaper Writings. August 1831-October 1834. Part II. 1986.

"Prospects of France. VIII", Examiner (abril 10, 1831). En CW, Vol. XXII.

"Rationale of Representation", London Review (julio 1835). En CW, Vol. XVIII. Essays on Politics and Society. Part I. 1977.

"Sedgwick's Discurse", London Review (abril 1835). En CW, Vol. X. Essays on Ethics, Religion and Society. 1985.

"State of Parties in France", Examiner (28 de agosto 1831). En CW, Vol. XXIII. 
“The Earlier Letters of John Stuart Mill 1812-1848. Part I". En CW, Vol. XII. 1963.

"The English National Character". Montly Repository, junio 1834. En CW, Vol. XXIII: Newspaper Writings (August 1831-October 1834). Part II. 1986.

“The Earlier Letters of John Stuart Mill 1812-1848. Part II". En CW, Vol. XIII. 1963.

“The Latter Letters of John Stuart Mill 1849-1973. Part I". En CW, Vol. XIV. 1972.

“The Latter Letters of John Stuart Mill 1849-1973. Part II”. En CW, Vol. XV. 1972.

“The Latter Letters of John Stuart Mill 1849-1973. Part III". En CW, Vol. XVI. 1972.

“The Negro Question”, Fraser's Magazine (enero de 1850). En CW, Vol. XXI.

"The Peerage Question in France", Examiner (septiembre 4, 1831). En $C W$, Vol. XXIII.

The Principles of Political Economy with Some of Their Applications to Social Philosophy. En CW, Vol. II-III. 1965.

"The Spirit of the Age", Examiner (enero a mayo 1831). En $C W$, Vol. XXII. Newspaper Writings. December 1822-July 1831. Part I. 1986.

- The Subjection of Women. En CW, Vol. XXI. Essays on Equality, Law and Education. 1984.

"The Universities". En CW, Vol. XXVI. Journals and Debating Speeches. Part I. 1988.

"Vindication of the French Revolution of February 1848", Westminster Review (abril 1849). En CW, Vol. XX. Essays on French History and Historians. 1985.

Utilitarism. En CW, Vol. X. Essays on Ethics, Religion and Society. 1985.

Pollitzer, M. "Individuos Perdidos en la Multitud: Mill y Tocqueville sobre la Sociedad Democrática". Revista de Instituciones, Ideas y Mercados 56, 2012, pp. 5-34.

Rousseau, J. J. Discurso Acerca del Origen de la Desigualdad entre los Hombres [1755]. Buenos Aires: Ateneo 2001.

Varouxakis, G. "National Character in J. S. Mill's Thought". History of European Ideas 24, 1998, pp. 375-391.

- "Guizot's History Works and John Stuart Mill's Reception of Tocqueville”. History of Political Thought Vol. 20 (2), 1999, pp. 292312. 